\title{
A NEW SOLUTION FOR LINEAR DIFFERENCE EQUATIONS
}

\author{
WOLFGANG J. STERNBERG
}

We shall give a solution of the fundamental difference equation:

$$
F(t+1)-F(t)=\phi(t) .
$$

As to our method, we stress that the theory of Fourier series is used. Accordingly, the variable $t$ is assumed to be real. We suppose that $\phi(t)$ is integrable and has bounded variation in every finite interval of $t$ or satisfies any other condition sufficient for expansion in a Fourier series. For simplicity we at first assume that $\phi(t)$ is continuous. Our solution is

(2) $\quad F(t)=-\frac{\phi(t)}{2}+\int_{a}^{t} \phi(\tau) d \tau+2 \sum_{k=1}^{\infty} \int_{a}^{t} \phi(\tau) \cos 2 \pi k(t-\tau) d \tau$,

where $a$ is constant.

The above series is not a Fourier series in the usual sense, since the upper limit of the integrals is not constant, but the variable $t$ itself.

To prove the truth of our statement we compute the difference

$$
\begin{aligned}
F(t+1)-F(t)= & -\frac{\phi(t+1)-\phi(t)}{2}+\int_{t}^{t+1} \phi(\tau) d \tau \\
& +2 \sum_{k=1}^{\infty} \int_{t}^{t+1} \phi(\tau) \cos 2 \pi k(t-\tau) d \tau
\end{aligned}
$$

Now, expansion of $\phi(t)$ in a Fourier series in any interval of length 1, say in the interval $c \cdots c+1, c$ meaning an arbitrary real constant, gives

$$
\phi(t)=\int_{c}^{c+1} \phi(\tau) d \tau+2 \sum_{k=1}^{\infty} \int_{c}^{c+1} \phi(\tau) \cos 2 \pi k(t-\tau) d \tau .
$$

The series represents $\phi(t)$ in the interior of the said interval, but it represents the value $(\phi(c)+\phi(c+1)) / 2$ at either end point, say at the left end point $c$. Therefore

$$
\frac{\phi(c)+\phi(c+1)}{2}=\int_{c}^{c+1} \phi(\tau) d \tau+2 \sum_{k=1}^{\infty} \int_{c}^{c+1} \phi(\tau) \cos 2 \pi k(c-\tau) d \tau .
$$

This holds for every $c$ and we can write $t$ instead of $c$, giving

Presented to the Society, February 26, 1944, under the title On difference equations; received by the editors October 17, 1944. 
(4) $\frac{\phi(t)+\phi(t+1)}{2}=\int_{t}^{t+1} \phi(\tau) d \tau+2 \sum_{k=1}^{\infty} \int_{t}^{t+1} \phi(\tau) \cos 2 \pi k(t-\tau) d \tau$.

From (3) and (4) it follows immediately that

$$
F(t+1)-F(t)=-\frac{\phi(t+1)-\phi(t)}{2}+\frac{\phi(t)+\phi(t+1)}{2}=\phi(t) .
$$

Now we allow $\phi(t)$ to become discontinuous. We suppose that at every point of discontinuity the limits $\phi(t+0)$ and $\phi(t-0)$ exist, which is certainly true in case of functions of bounded variation, and that the relation

$$
\frac{\phi(t+0)+\phi(t-0)}{2}=\phi(t)
$$

holds. The solution is then given by

$\left(2^{*}\right) F(t)=-\frac{\phi(t-0)}{2}+\int_{a}^{t} \phi(\tau) d \tau+2 \sum_{k=1}^{\infty} \int_{a}^{t} \phi(\tau) \cos 2 \pi k(t-\tau) d \tau$.

It follows indeed that

$$
\begin{aligned}
F(t+1)-F(t)= & -\frac{\phi(t+1-0)}{2}+\frac{\phi(t-0)}{2} \\
& +\int_{t}^{t+1} \phi(\tau) d \tau+2 \sum_{k=1}^{\infty} \int_{t}^{t+1} \cdots
\end{aligned}
$$

But the series on the right represents now the value

$$
\begin{aligned}
\int_{t}^{t+1} \phi(\tau) d \tau+2 \sum_{k=1}^{\infty} \int_{t}^{t+1} \phi(\tau) & \cos 2 \pi k(t-\tau) d \tau . \\
= & \frac{\phi(t+0)+\phi(t+1-0}{2} .
\end{aligned}
$$

Therefore, considering (5),

$$
F(t+1)-F(t)=\frac{\phi(t-0)+\phi(t+0)}{2}=\phi(t) .
$$

Plana (1820) and, independent of him, Abel (1823) were the first to solve equation (1). Their starting point was the Euler's "sum formula," and they found the same solution

$$
F(t)=-\frac{\phi(t)}{2}+\int^{t} \phi(\tau) d \tau+2 \int_{0}^{\infty} \frac{q(t, \tau)}{e^{2 \pi \tau}-1} d \tau
$$


where

$$
q(t, \tau)=[\phi(t+\tau i)-\phi(t-\tau i)] / 2 i \text { and } i=(-1)^{1 / 2}
$$

and the lower limit of the first integral is a constant. The above solution is not identical with our solution, as given by (2), since, although the first two terms agree, the third ones do not. Take, for instance, $\phi(t) \equiv 1$. Then $q(t, \tau) \equiv 0$, and the integral

$$
\int_{0}^{\infty} \frac{q(t, \tau)}{e^{2 \pi \tau}-1} d \tau
$$

vanishes identically in $t$, but the series

$$
\sum_{k=1}^{\infty} \int_{a}^{t} \cos 2 \pi k(t-\tau) d \tau
$$

does not.

The proofs of Plana and Abel were not exact. Only Cauchy gave (1826) an exact derivation of the above solution, applying the theory of functions of a complex variable and, in particular, his calculus of residues. For more details see Ernst Lindelöf, Le calcul des residues, Paris, 1905, p. 68.

Later on H. Poincaré established the concept of asymptotic representation of a function and applied it to difference equations, Amer. J. Math. vol. 7 (1885) pp. 213-217 and 237-258. Out of several more recent authors we name in particular N. E. Nörlund, R. D. Carmichael and G. D. Birkhoff. They proved, by using different methods, the existence of analytic solutions of linear difference equations and investigated their properties.

We finally mention two books: Nörlund, Vorlesungen über Differenzenrechnung, Berlin, Julius Springer, 1924, which includes a very extended list of the literature, and P. M. Batchelder, An introduction to linear difference equations, Cambridge, Mass., The Harvard University Press, 1927, which presents the methods of both Nörlund and Birkhoff, and moreover gives some important new results.

CORNELl UNIVERSITY 